\title{
Structural Design Using Simulation Based Reliability Assessment
}

\author{
S. Vukazich, P. Marek
}

The concept of Simulation Based Reliability Assessment (SBRA) for Civil Engineering structures is presented. SBRA uses bounded histograms to represent variable material and geometric properties as well as variable load effects, and makes use of the Monte Carlo technique to perform a probability-based reliability assessment. SBRA represents a departure from the traditional deterministic and semi-probabilistic design procedures applied in codes and as such requires a "re-engineering" of current assessment procedures in accordance with the growing potential of computer and information technology. Three simple examples of how SBRA can be used in Structural Engineering design are presented: 1) the reliability assessment of a steel beam, 2) a truss bar subjected to variable tension and compression, and 3) the pressure on the wall of an elevated water tank due to earthquake load.

Keywords: Structural Engineering, Reliability Assessment, Monte Carlo Method.

\section{Introduction}

The idea of structural reliability can be very simply stated: the structure must have greater resistance than the load effects on the structure demand. The resistance of a structure is a variable quantity depending on material strengths, accuracy of analytical models, geometric tolerances, and many other factors. Similarly, load effects due to wind, seismic, vehicle and other loads are variable. The first reliability assessment concepts used for design were deterministic and based on experience, using single safety factors to account for uncertainties in both resistance and load effects. Since the early 1960's many national and international design codes have been moving in accordance with a limit states design philosophy based on the partial factors concept in which the variability of resistance and load effect combinations are analyzed separately. This approach allows for taking more precisely into account the strength of the structure beyond the linear elastic limit, second order effects, and the accumulation of damage.

In partial factors design, nominal values of loads (or load effects) are chosen and combinations of these loads are specified for design using special coefficients and load factors that multiply the nominal loads in order to define design values that take into account load combinations. The resistance of the structure or element of the structure is represented by its ultimate strength. The ultimate strength is multiplied by a resistance factor to take into account its variability. Structural safety is achieved when the factored resistance equals or exceeds the factored load effect combination.

Unfortunately, when considering the multi-random variable input which governs both resistance and loading, the analytical mathematical solutions required to determine the design point can become extremely difficult, if not impossible. There is also some question as to the appropriateness of using ultimate (i.e. collapse) behavior as the reference level for reliability assessment. Thus, load and resistance factors have been chosen based on years of design experience coupled with experimental results and calibration. Because of the large amount of design experience associated with current design codes, reliable designs for many classes of structures can be realized, but as a consequence, codes based on the partial reliability factors concept remain, from the designer's point of view, deterministic in nature.

\section{Simulation based reliability assessment (SBRA)}

An alternative approach, which is now possible due to modern computer technology, is to use Monte Carlo simulation to model the variability of both load effects and resistance. Simulation Based Reliability Assessment (SBRA) as presented by Marek et al [1] allows for detailed evaluation of individual load effects and their combinations. All loads are random in nature and can be described as random variables. In this approach, individual loads are represented by load duration curves and corresponding bounded (non-parametric) histograms and not by nominal values and load factors as is the case in many current codes. Similarly, variable quantities (e.g. yield strength) that affect the resistance of a structure or an element can also be expressed in terms of bounded histograms.

Fig. 1 is an outline of how a bounded histogram that represents a loading action may be constructed. In Fig. 1(a) the time history of an action $\mathrm{F}(\mathrm{t})$ is plotted. In this particular case, the action represents a long lasting load on a structure. Fig. 1(b) shows the time history sorted so that the minimum intensity is on the left and orders the intensities ending with the maximum intensity on the right. This sorted time history is called the load duration curve. The load duration curve can be transformed into the bounded histogram shown in Fig. 1(c). In SBRA all loads are represented as bounded histograms and can be constructed from data on load history.

Another key element in SBRA is the definition of a reference level to define the limit of the "usefulness" of a structure or element. Any exceedance of this "usefulness" limit would impair the ability of the structure or elements of the structure to perform "safely" and would require replacement or repair. Obviously, these limits may have a different character depending on the type of structure, material, loading, etc. For example, in the case of simple strength, the limit resistance may be set as the onset of yielding or to some tolerable magnitude of permanent plastic deformation. In the case of fatigue, 


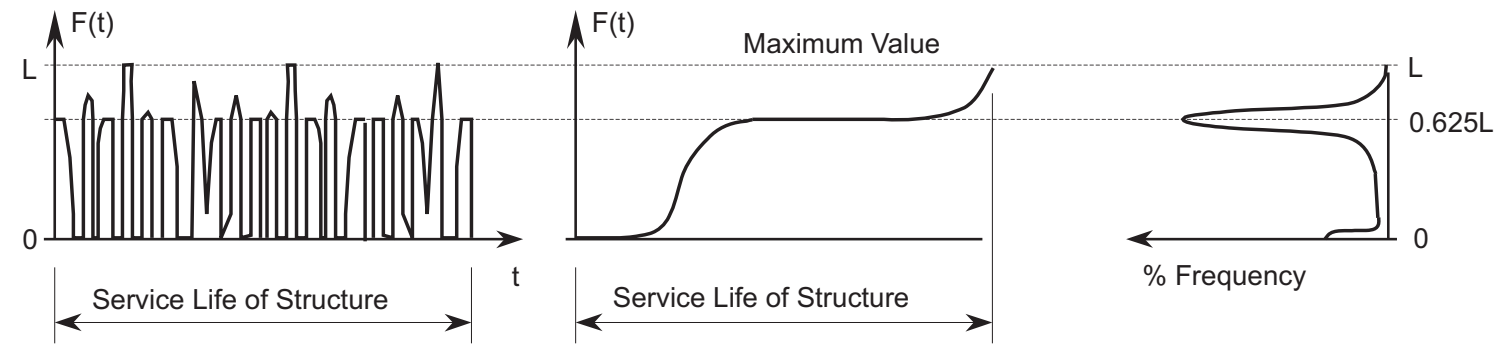

$\begin{array}{lll}\text { a) Load Time History } & \text { b) Sorted Load Time History: Load Duration Curve } & \text { c) Bounded Histogram }\end{array}$

Fig. 1: Generation of load duration curve and corresponding bounded histogram for long lasting load

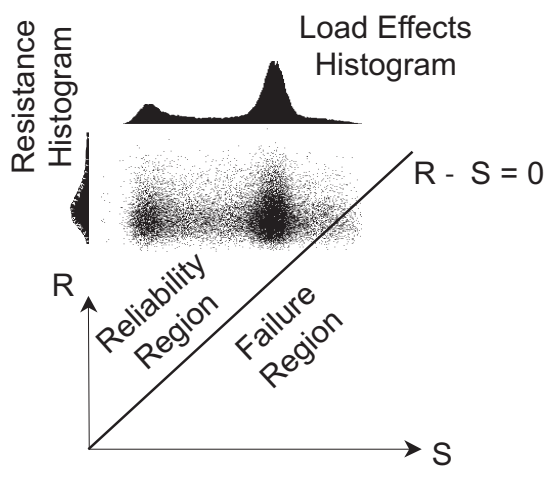

Fig. 2: Graphical representation of simulation based reliability assessment

the limiting value may be set as a tolerable magnitude of accumulated damage or by a tolerable length of fatigue crack.

Fig. 2 is an illustration of how structural reliability can be checked using simulation from Marek et al [1]. The method uses direct Monte Carlo Simulation to determine the probability of failure corresponding to any combination of variable load effects, S, and resistance, $\mathrm{R}$. The required number of computer simulations are performed according to the distributions of $\mathrm{R}$ and $\mathrm{S}$. To obtain the probability of failure, the number of simulations (each "dot" in Fig. 2 corresponding to the interaction of $\mathrm{R}$ and $\mathrm{S}$ ) in the Failure Region (the region to the right of the $\mathrm{R}-\mathrm{S}=0$ line in Fig. 2) is divided by the total number of simulations. To check the design, the result- ing probability of failure is compared to an acceptable target probability.

The following three examples illustrate how SBRA can be used in structural design. The first example examines the safety and serviceability assessment of a steel beam. The second example presents the assessment of a diagonal member of a steel truss subjected to loading that can induce both tension and compression in the member. The third example is an illustration of how SBRA can be used in earthquake engineering.

\section{Example 1: reliability of a steel beam}

The reliability of the simply supported steel beam shown in Fig. 3 exposed to the uniform dead load $q$, long-lasting point load $F_{1}$ and short-lasting point loads $F_{2}$ will be examined. Lateral-torsional buckling of the beam is prevented, only the elastic response to the loading is considered, and

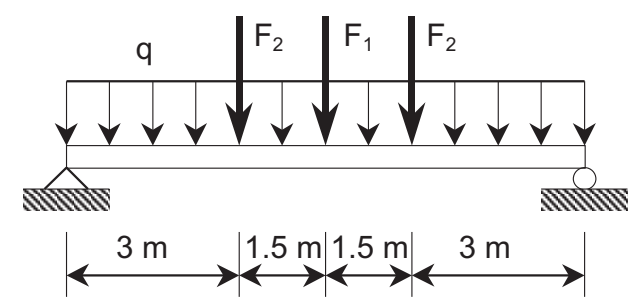

Fig. 3: Steel beam example

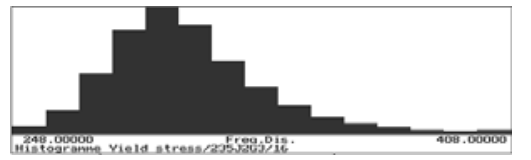

a) Yield Stress (S235_15.his)

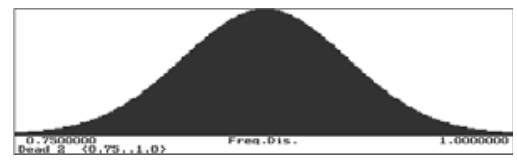

b) Dead Load (Dead2.his)

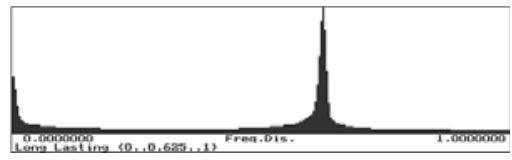

c) Long Lasting Load (Long1.his)

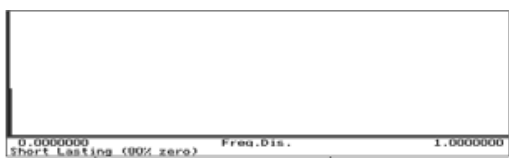

d) Short Lasting Load (Short1.his)

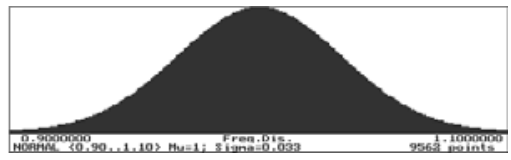

e) Moment of Inertia (N1-10.his)

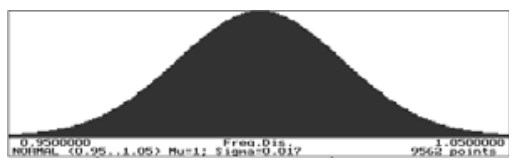

f) Section Modulus (N1-05.his)

Fig. 4: Bounded histograms used to represent variable loads and properties 
effects of residual stresses are neglected. The target probabilities $P_{\mathrm{d}}=0.00007$ for safety and $P_{\mathrm{d}}=0.07$ for serviceability are considered.

The steel is grade $\mathrm{S} 235$, while the extreme load values are dead load, $q=5 \mathrm{kN} / \mathrm{m}$; long lasting load, $F_{1}=75 \mathrm{kN}$; and short-lasting load, $F_{2}=30 \mathrm{kN}$. The nominal beam span is $L=9 \mathrm{~m}$ and the rolled section properties are: nominal section modulus $W_{\text {nom }}=1147500 \mathrm{~mm}^{3}$, and the nominal moment of inertia $I_{\text {nom }}=329296875 \mathrm{~mm}^{4}$.

The yield stress $F_{\mathrm{y}}$ is expressed by the bounded histogram S235_16.his shown in Fig. 4(a). The load variations are represented by the histograms Dead2.his, Long1.his, and Short1.his shown in Figs. 4(b), 4(c), and 4(d). The variations of the geometric properties of the section due to over and under-rolling are expressed by normal distributions with a range of $\pm 10 \%$ for $I_{\text {nom }}$, represented by histogram N1-10.his shown in Fig. 4(e), and similarly, a $\pm 5 \%$ range for $W_{\text {nom }}$, expressed by the histogram N1-05.his shown in Fig. 4(f). The beam length $L$ is taken to be constant.

Variable quantities are represented by multiplying the extreme or characteristic value by the corresponding histogram. For example, the variation of the section modulus is represented by

$$
W_{\text {var }}=W_{\text {nom }} *(\text { histogram N1-05). }
$$

Note that in all subsequent equations, the subscript var is used to represent a variable quantity.

\subsection{Reliability assessment for safety}

In this example, the reference level applied in the calculation of probability of failure $P_{\mathrm{f}}$ is defined by the onset of yielding with the prescribed target probability of $P_{\mathrm{d}}=0.00007$.

The load effect combination at the critical section of the beam can be expressed as:

$$
S=q_{\mathrm{var}} L^{2} / 8+F_{1 \mathrm{var}} L / 4+F_{2 \mathrm{var}} L / 3 .
$$

The reliability function at the critical section can be expressed as:

$$
R=0.9 F_{\text {y var }} W_{\text {var }} .
$$

Where the 0.9 reduction factor is used to represent the difference between the reference level yield stress and the experimentally derived yield stress $F_{\mathrm{y}}$. A more detailed discussion of reference levels may be found in Marek et al [1].

The Safety function is defined as:

$$
S F=R-S .
$$

Using the M-Star program, developed by Marek et al [1], the Safety Function can be evaluated and the output is shown in Fig. 5(a). A total of 100000 simulation steps were used. From the M-Star output, one can see that the probability of failure of this section (i.e. the probability that the Safety Function is zero or less) is 0.00006 . Since the probability of failure is less than the target probability $(0.00006<0.00007)$ the safety of the section is adequate.

\subsection{Reliability assessment for serviceability}

Next the reference serviceability limitation will be checked. The maximum tolerable deflection is defined by the limit of $L / 350$. The prescribed corresponding target probability is $P_{\mathrm{d}}=0.07$.
The deflection referring to the variable load effect combination is:

$$
\delta=\frac{1}{E I_{\mathrm{var}}}\left(\frac{5 L^{4}}{384} q_{\mathrm{var}}+\frac{L^{3}}{48} F_{1 \mathrm{var}}+0.0355 L^{3} F_{2 \mathrm{var}}\right) .
$$

The Serviceability Function is defined by:

$$
S F=\frac{L}{350}-\delta .
$$

The M-Star program output for the serviceability analysis is shown in Fig. 5(b). A total of 100000 simulation steps were used. From the M-Star output, one can see that the probability of failure of this section (i.e. the probability that the Serviceability Function is zero or less) is 0.00581. Since

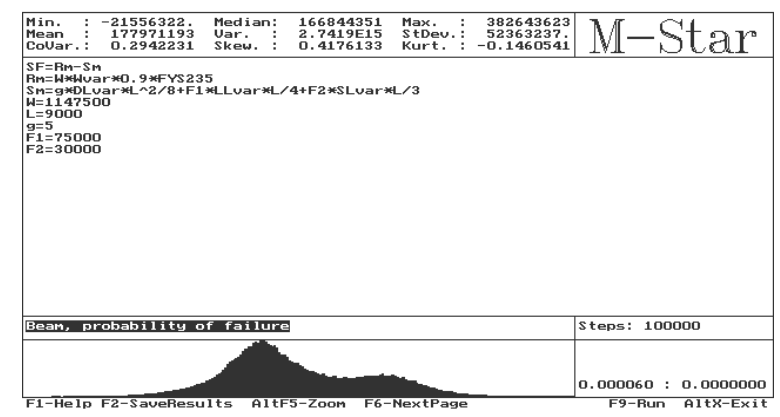

a) Reliability Analysis for Safety

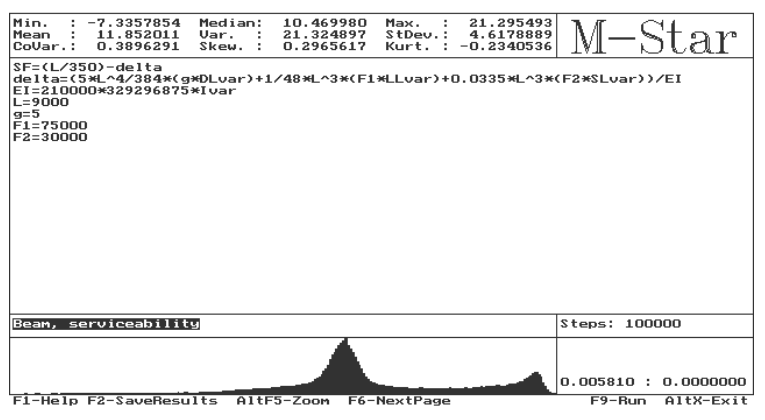

b) Reliability Analysis for Serviceability

Fig. 5: M-Star ${ }^{\mathrm{TM}}$ program output for reliability assessment

the probability of failure is less than the target probability $(0.00581<0.07)$ the serviceability of the section is adequate.

\section{Example 2: truss bar subjected to tension and compression}

The pin-connected truss shown in Fig. 6 is exposed to horizontal and vertical variable mutually uncorrelated loads $W L, S L$ and $D L$ acting in the plane of the truss. Translation of the joints out of the plane of the truss is prevented. This example illustrates how simulation based reliability assessment can be used to assess the diagonal bar "a" indicated in Fig. 6 to resist variable tension and compression due to the loads. Initial eccentricity is considered due to fabrication and also to express the effect of residual stresses. All load effects and variables are considered to be statistically independent. The target probability used for reliability assessment for safety 
Table 1: Characteristic values of applied loads corresponding to load factors

\begin{tabular}{|l|c|c|c|c|}
\hline \multicolumn{1}{|c|}{ Loading } & Characteristic Value [kN] & Eurocode 1993 Load Factors & Extreme Values [kN] \\
\hline Dead DL & 400.0 & 1.35 & 1.00 & $540.0,350.0$ \\
\hline Short lasting SL & 1200.0 & 1.50 & 0.00 & $1800.0,0.0$ \\
\hline Wind WL & 200.0 & 1.50 & -1.50 & $300.0,-300.0$ \\
\hline
\end{tabular}

Table 2: Geometric and material properties of selected rolled steel shapes

\begin{tabular}{|c|c|c|}
\hline Rolled Shape & IPE 330 & IPE 360 \\
\hline Cross-sectional area A $\left[\mathrm{m}^{2}\right]$ & 0.00626 & 0.00727 \\
\hline Radius of gyration $\mathrm{r}[\mathrm{m}]$ & 0.03547 & 0.03782 \\
\hline Steel grade (ČSN 73 1401, ČSN P ENV 1993-1-1) & Fe360/S235 & Fe360/S235 \\
\hline
\end{tabular}

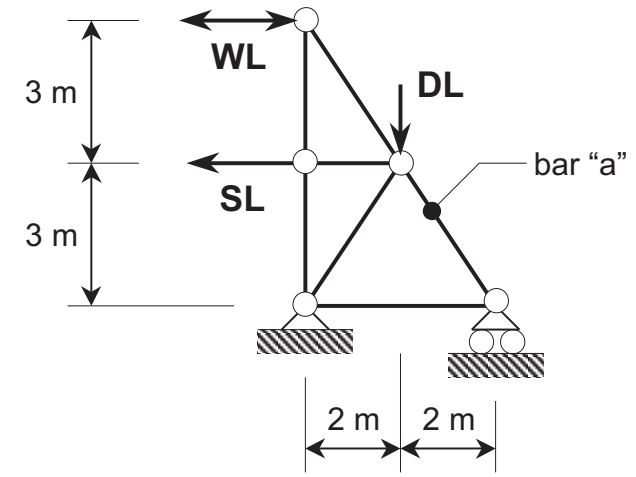

Fig. 6: Geometry of the example truss

considering tension and buckling in this example is assumed to be $P_{\mathrm{d}}=0.00007$.

Table 1 contains the characteristic values of the loads and corresponding load factors according to Eurocode 1993 (further details can be found in [4]). The nominal geometrical and mechanical properties of the rolled steel shapes are shown in Table 2.

The bounded histograms used to represent the variable quantities in this example are shown in Fig. 7. All variable quantities are expressed in a similar manner as presented in the previous example.

The resistance in tension $R_{\mathrm{t}}$ of the truss bar is defined as:

$$
R_{t}=0.9 F_{y \text { var }} A_{\text {var }} .
$$

The resistance $R_{\mathrm{c}}$ in compression can be defined by the following equation from Marek et al [1], [4]:

$$
\begin{aligned}
R_{c}=\frac{R_{1}-\sqrt{R_{1}^{2}-\frac{4(L / r)^{2}}{\pi^{2} E A_{\mathrm{var}}} F_{y \mathrm{var}} A_{\mathrm{var}}}}{\left(\frac{2(L / r)^{2}}{\pi^{2} E A_{\mathrm{var}}}\right)} ; \\
\text { where } \quad R_{1}=1+\frac{F_{y \mathrm{var}}(L / r)^{2}}{\pi^{2} E}+\frac{L e_{\mathrm{var}}\left(1+0.1 s_{\mathrm{var}}\right) c}{r^{2}} .
\end{aligned}
$$

Where the constant quantities in Eqn. 8 are: $E$, the elastic modulus; $c$, the distance to the extreme fibers from the centroidal axis; $L$, the length of the bar; and $r$, the radius of gyration. The variable quantities are: $s_{\mathrm{var}}$, a variable parameter that takes into account residual stress in the cross section (Fig. 7(d)); $e_{\mathrm{var}}$, the initial eccentricity (Fig. 7(e)); and $A_{\mathrm{var}}$, the cross sectional area (Fig. 7(f)).

The reliability assessment according to SBRA is based on the analysis of the Safety Function:

$$
S F=R-S
$$

where $R$ is the resistance (referring to the onset of yielding or buckling of the truss bar) and $S$ is the variable load effect

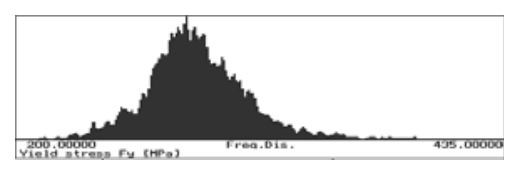

a) Yield Stress (T235fy01.his)

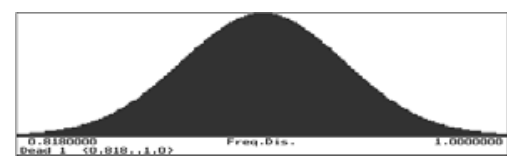

b) Dead Load (Dead1.his)

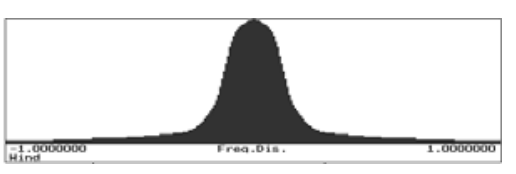

c) Wind Load (Wind1.his)

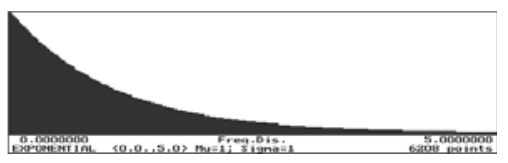

d) Residual Stress (Expon1.his)

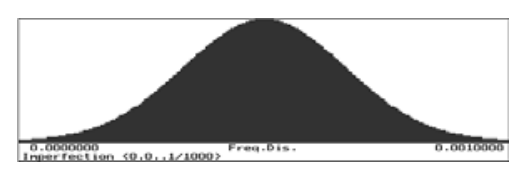

e) Initial Eccentricity (Imp1000.his)

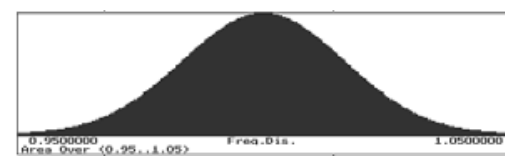

f) Cross Sectional Area (Area-M.his)

Fig. 7: Bounded histograms used to represent variable loads and variable properties 

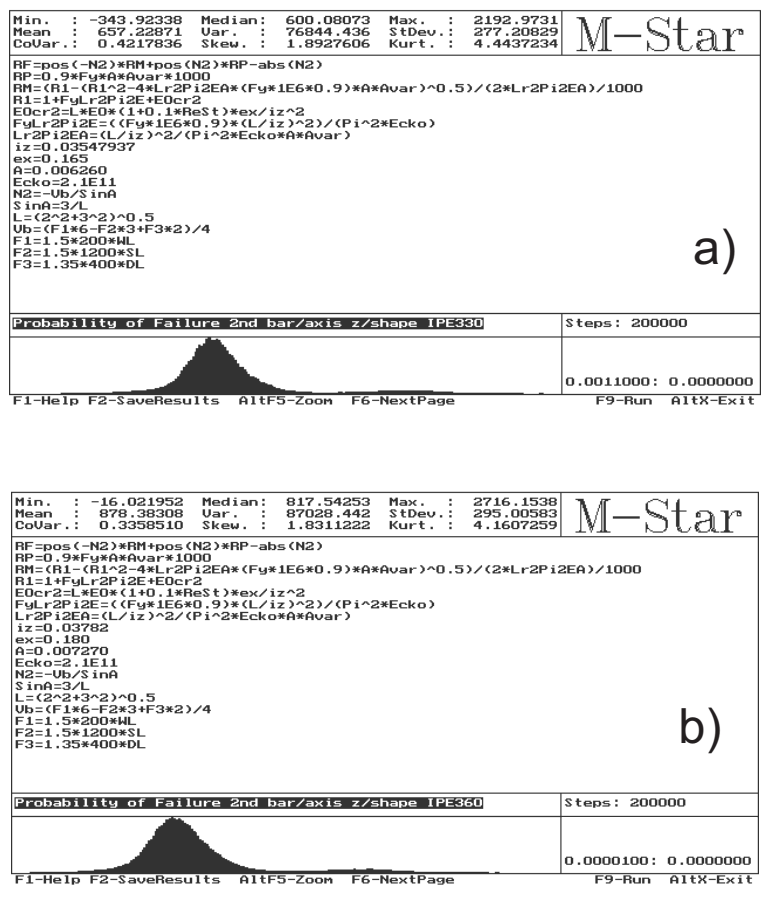

Fig. 8: M-Star ${ }^{\mathrm{TM}}$ program output: a) IPE330, b) IPE360

expressed by the axial force in the truss bar (variable $N 2$ in the M-Star and Anthill analyses).

Two possible sections are considered for the truss bar. The first is an IPE 330 section with the probability of failure $P_{\mathrm{f}}$ determined using SBRA and the M-Star program to analyze the Safety Function with the output shown in Fig. 8(a). As illustrated previously in Fig. 2, the probability of failure can also be determined by plotting Resistance ( $R$ ) versus axial load in the truss bar (N2). This analysis was performed using the Anthill program (developed by Marek et al [1]) with the output shown in Fig. 9(a). Note that 200000 simulation steps were used and both the M-Star and Anthill analy-
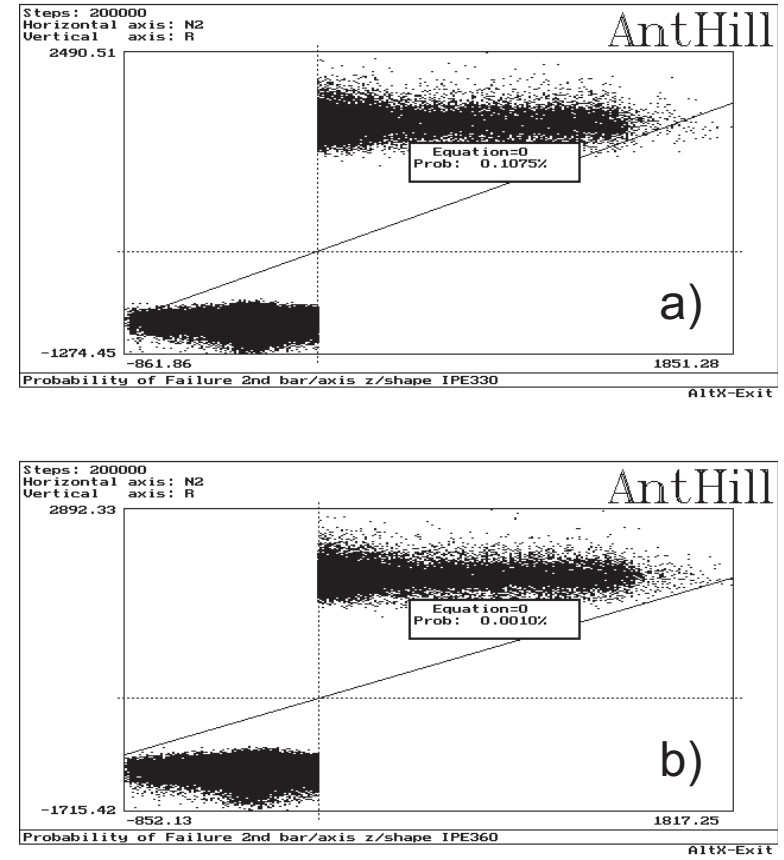

Fig. 9: Anthill ${ }^{\mathrm{TM}}$ program output: a) IPE330, b) IPE360

ses yield a similar probability of failure, $P_{\mathrm{f}}=0.00110$ and $P_{\mathrm{f}}=0.001075$, respectively. From both the M-Star and the Anthill output, we can see that the IPE 330 section is not adequate $\left(P_{\mathrm{f}}=0.0011>P_{\mathrm{d}}=0.00007\right)$.

The second analysis considers a larger IPE 360 section and results in a lower probability of failure $\left(P_{\mathrm{f}}=0.00001\right.$ from both the M-Star analysis and the Anthill analysis). The M-Star and Anthill output for the IPE 360 section is shown in Figs. 8(b) and 9(b), respectively. From the analyses, one can see that the IPE 360 section is adequate to resist both the tension and the compression induced by the applied loads $\left(P_{\mathrm{f}}=0.00001<\mathrm{P}_{\mathrm{d}}=0.00007\right)$.

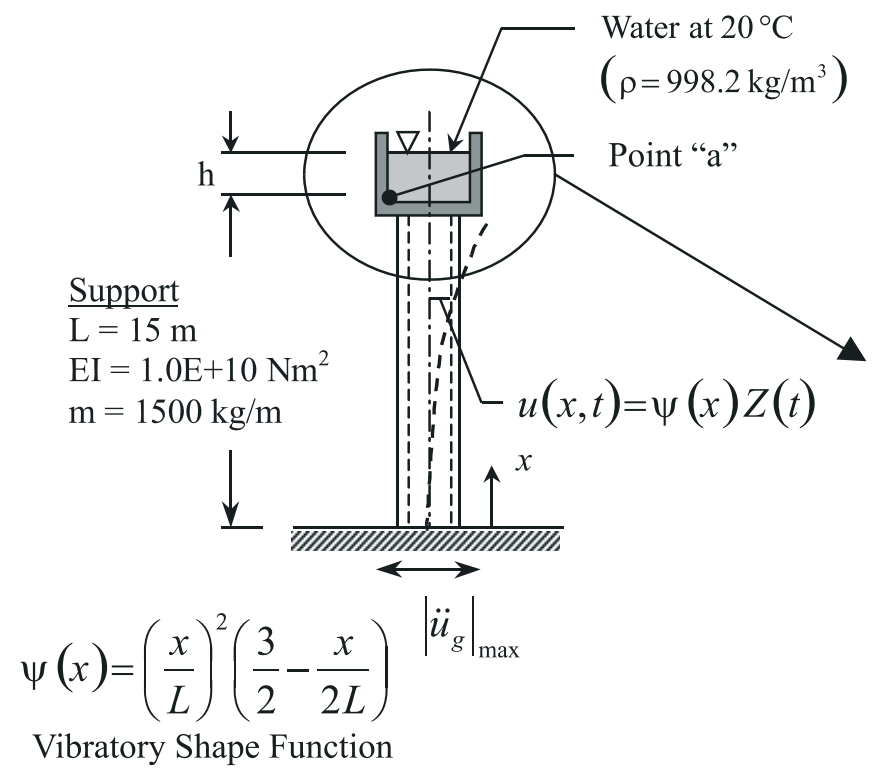

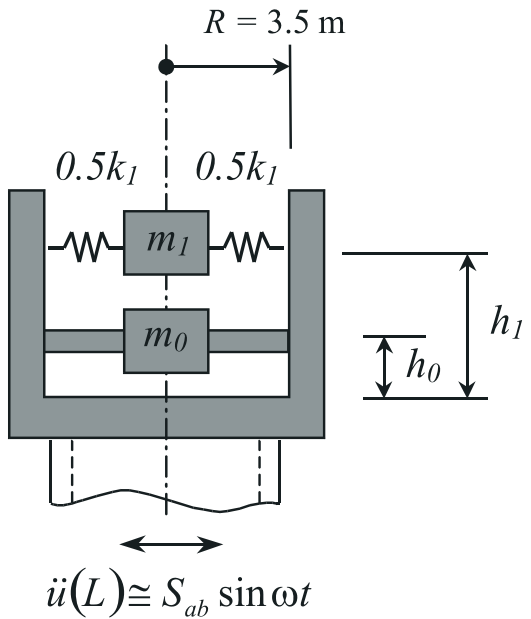

Effective Mass-Spring System

Fig. 10: Elevated water tank structure and physical model of water in the tank 


\section{Example 3: pressure on the wall of an elevated water tank due to earthquake}

In this example, the maximum pressure on the wall of the cylindrical elevated water tank shown in Fig. 10 is calculated for earthquake loading. The effect of the variation of water level, $h$, and other variable properties on the maximum pressure exerted on the wall at point " $a$ " is studied. The characteristic values of the mass and stiffness of the tank support are shown in Fig. 10. The vibratory response of the entire

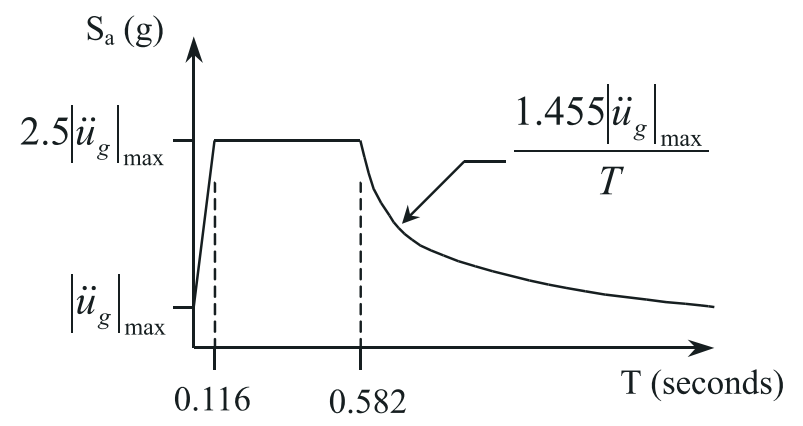

Spectrum 1. Structure on Stiff Soil Support

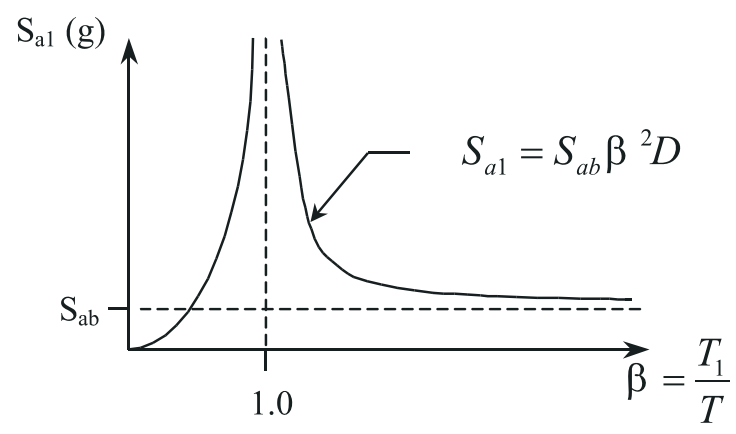

Spectrum 2. Water in the Tank

Fig. 11: Response spectra for the elevated tank problem

structure is approximated by the shape function $\psi(x)$. The dynamic properties of the water in the tank are represented by the effective spring-mass system shown in Fig. 10. The mass, $m_{1}$, and the stiffness, $k_{1}$, represent the oscillation of the water in the tank due to acceleration of the tank base (Harris [6]). The effect of tank rotation and the rotational inertia of the tank are negligible. The maximum peak ground acceleration at the site is taken to be $0.44 \mathrm{~g}$, with a variation of $\pm 10 \%$.
The response spectra given in Fig. 11 are representative of the response of the entire structure (Spectrum 1) and the equivalent mass-spring system for the water in the tank (Spectrum 2).

The variable quantities considered in this problem are peak ground acceleration, tank radius, water height in the tank, distributed mass of the tank support structure, and the bending stiffness of the support structure. Each of these variables is represented by multiplying the characteristic value by a bounded, normal distribution histogram. The characteristic and extreme values of the variable quantities are summarized in Table 3 .

Note that for this illustrative example, the response of the entire tank structure and the equivalent spring-mass system that represents the effect of the oscillation of the water in the tank are considered separately. This approach can be used for the ranges of stiffness and mass in this problem. In more general cases, the analysis should consider the dynamics of the entire coupled system. The entire elevated tank structure is modeled as an equivalent Single Degree of Freedom (SDOF) system based on the shape function given in Fig. 10. In this manner, the deformation of the tank can be expressed as:

$$
u(x, t)=\psi(x) Z(t) .
$$

Using Eqn. 10, the equivalent SDOF equation of motion for a structure subjected to the earthquake ground acceleration $\ddot{u}_{\mathrm{g}}$ can be written as:

$$
Z+2 \xi \omega Z+w^{2} Z=\left(\Gamma / m^{*}\right) \ddot{u}_{\mathrm{g}}
$$

where for this problem and the defined shape function:

$$
\begin{aligned}
& \xi=\text { damping ratio }(5 \%) \\
& \text { ported tank structure } \\
& m^{*}=0.2357 m L+M \\
& k^{*}=3 E I / L^{3} \\
& \Gamma=0.375 m L+M \\
& k_{1}=\text { equivalent stiffness of fluid in the tank } \\
& m=\text { distributed mass of the support structure } \\
& M=\text { mass of the supported tank and water }
\end{aligned}
$$$$
\omega=\sqrt{k^{*} / m^{*}}=\text { fundamental circular frequency of the sup- }
$$

Calculation details for an equivalent SDOF system can be found in any structural dynamics text (Clough and Penzien [5]). The maximum acceleration response at the level of the tank $(x=L)$ can be found using Spectrum 1 in Fig. 11 and the fundamental period, $T$, of the elevated tank structure:

$$
S_{\mathrm{ab}}=\ddot{u}(L)_{\max }=\psi(L) \frac{\Gamma}{m^{*}} S_{\mathrm{a}}=\frac{\Gamma}{m^{*}} S_{\mathrm{a}} .
$$

Table 3: Variable quantities for tank pressure example

\begin{tabular}{|l|c|c|}
\hline \multicolumn{1}{|c|}{ Load/Property } & Characteristic value & Extreme values \\
\hline Peak ground acceleration & $0.440 \mathrm{~g}$ & $0.396 \mathrm{~g}, 0.484 \mathrm{~g}$ \\
\hline Tank radius & $3.5 \mathrm{~m}$ & $3.15 \mathrm{~m}, 3.85 \mathrm{~m}$ \\
\hline Water Height & $3.0 \mathrm{~m}$ & $2.0 \mathrm{~m}, 4.0 \mathrm{~m}$ \\
\hline Mass of support structure & $1500.0 \mathrm{~kg} / \mathrm{m}$ & $1350.0 \mathrm{~kg} / \mathrm{m}, 1650.0 \mathrm{~kg} / \mathrm{m}$ \\
\hline Bending stiffness of support & $1.0 \mathrm{E}+10 \mathrm{Nm}^{2}$ & $0.9 \mathrm{E}+10 \mathrm{Nm}^{2}, 1.1 \mathrm{E}+10 \mathrm{Nm}^{2}$ \\
\hline
\end{tabular}


The maximum displacement response of the oscillating water in the tank can now be found by assuming that the acceleration at the base of the tank can be taken as approximately sinusoidal as shown in Fig. 10:

$$
\ddot{u}(L) \cong S_{\mathrm{ab}} \sin \omega t
$$

where $\omega$ is the circular frequency of the entire tank structure. Spectrum 2 shown in Fig. 11 is the result of assuming that the acceleration at the base of the tank is sinusoidal and the maximum acceleration can be written as:

$$
S_{\mathrm{a} 1}=S_{\mathrm{ab}} \beta^{2} D
$$

where:

$\beta=\omega / \omega_{1}=T_{1} / T=$ frequency ratio of the tank structure and water

$D=\frac{1}{1-\beta^{2}}$ for an undamped system.

Details of the derivation of Eqn. 14 can be found in Clough and Penzien [5]. The maximum acceleration at the base of the tank can be found from Eqns. 12 and 14 and the fundamental period of the water in the tank:

$$
T_{1}=2 p / \sqrt{k_{1} / m_{1}}
$$

where $k_{1}$ and $m_{1}$ are defined in Harris [6] as:

$$
m_{1}=M \frac{3}{5} \frac{\tanh \sqrt{\frac{27}{8}} \frac{h}{R}}{\sqrt{\frac{27}{8}} \frac{h}{R}} ; k_{1}=m_{1} \frac{g}{h} \sqrt{\frac{27}{8}} \frac{h}{R} \tanh \sqrt{\frac{27}{8}} \frac{h}{R} \text {. }
$$

The maximum displacement of the equivalent mass $m_{1}$ can be found by integrating the acceleration response:

$$
S_{\mathrm{d} 1}=S_{\mathrm{a} 1} / \omega^{2} \text {. }
$$

From the equivalent heights, $h_{0}$ and $h_{1}$, and from an analysis of the equivalent spring-mass system for the tank shown in Fig. 10 the pressure on the wall at depth $h$ can be expressed as:

$p_{\mathrm{w}}=S_{\mathrm{ab}} \rho h \frac{\sqrt{3}}{2} \tanh \sqrt{3} \frac{R}{h}+S_{\mathrm{d} 1} \rho R \frac{5}{8}\left(\frac{1}{\cosh \sqrt{\frac{27}{8}} \frac{h}{R}}\right)$

where $S_{\text {ab }}$ represents the maximum acceleration at the base of the tank and $S_{\mathrm{d} 1}$ represents the maximum displacement of the equivalent mass $m_{1}$ relative to the wall of the tank. Details of the derivation of Eqn. 18 can be found in Harris [6]. Note that Eqn. 18 conservatively assumes that both $S_{\mathrm{ab}}$ and $S_{\mathrm{d} 1}$ reach their maximum values at near the same instant in time.

The distribution of the pressure at the base of the tank wall $p_{\mathrm{w}}$ was calculated using the M-Star program using 100000 simulation steps. The M-Star output for this problem

Table 4: Variation of the maximum water pressure on the elevated tank wall

\begin{tabular}{|c|c|}
\hline Pressure at point "a” & Probability of non-exceedance \\
\hline $16547 \mathrm{~N} / \mathrm{m}^{2}$ & 0.0 (minimum) \\
\hline $26096 \mathrm{~N} / \mathrm{m}^{2}$ & 0.99 \\
\hline $27021 \mathrm{~N} / \mathrm{m}^{2}$ & 0.999 \\
\hline $27723 \mathrm{~N} / \mathrm{m}^{2}$ & 0.9999 \\
\hline $28516 \mathrm{~N} / \mathrm{m}^{2}$ & 1.0 (maximum) \\
\hline
\end{tabular}

is shown in Fig. 8 with a summary of the results given in Table 4.

A deterministic analysis for the water level at the maximum level of $h=4 \mathrm{~m}$ results in a pressure of $24727 \mathrm{~N} / \mathrm{m}^{2}$. From Fig. 12, the probability of the pressure exceeding $24727 \mathrm{~N} / \mathrm{m}^{2}$ is $=1-0.9057=0.0943(9.43 \%)$.

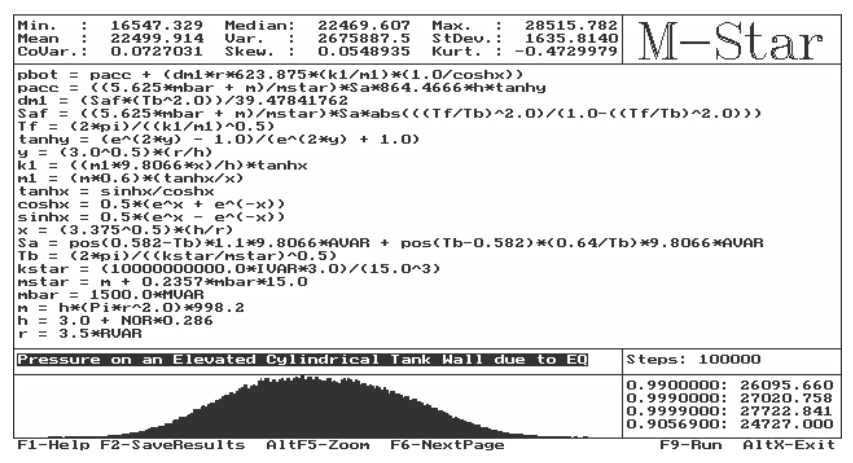

Fig. 12: Calculation of the maximum wall pressure using M-Star ${ }^{\mathrm{TM}}$

The results show that taking into account variable properties and the variation of the water level has a significant effect on the results and consequently on the design of the tank. In lieu of choosing normal distributions to describe variable quantities, historical water level records and collected data that describe other variable properties can be used for a more accurate analysis. Note also that this example was constructed for the purpose of illustrating SBRA, and so some of the simplifications and assumptions made may not be acceptable for all conditions. This analysis can easily be extended to include the probability of the occurrence of an earthquake.

\section{Conclusion}

The three examples presented only hint at the potential of SBRA as a tool for the designer. Many detailed examples of SBRA related to Civil Engineering design problems are presented in Marek et al [1], [3] and Brozzetti et al [2]. Unfortunately, at present, to include simulation based reliability assessment methods into codes and standards is not an easy task. In many ways current methods must be reexamined, in particular, the characterization of how variable loads are represented and distributed on structures. Since nearly all of the current design codes in Civil Engineering, from the designer's point of view, are deterministic in nature, assessment of an acceptable probability of failure is difficult. Considerable discussion by researchers, specification writing committees, and designers is needed to pave the way for innovation in Structural Engineering, starting with the transition from a deterministic to a probabilistic "way of thinking" (Marek et al [3]). Attention must be given to the "rules of the game" including the definition of the reference level in the probabilistic analysis of safety, durability, and serviceability. To date, the only code that allows for applying simulation and gives guidelines for target probability is the Structural Steel Design Code 73 1401-1998 (Appendix A) of the Czech National Standards. 
Computational methods like SBRA, allow designers to use, build, and update available data and knowledge bases to better represent the variable character of loading, material properties, and other factors in design and assessment. It is the opinion of the authors that SBRA takes advantage of modern information transfer and computer power to build a more general, accurate, consistent, and transparent representation of load and resistance, and represents the future of structural reliability assessment methods.

\section{Acknowledgements}

The results presented in this work were supported by the Leonardo da Vinci Agency (EC Brussels) and GACR 103/01/1410 and 103/96/K034 - Czech Republic.

Professor Ing. Ondřej Fischer, DrSc. of ITAM CAS, Academy of Sciences of Czech Republic, contributed to the development of Example 3 (see [3]).

\section{References}

[1] Marek, P., Guštar, M., Anagnos, T.: Simulation-based Reliability Assessment for Structural Engineers. CRC Press, Inc., Boca Raton, Florida, 1995

[2] Brozzetti, J., Guštar, M., Ivanyi, M., Kowalczyk, R., Marek, P.: TERECO-Teaching Reliability Concepts using Simulation Technique. Leonardo da Vinci Agency, EU, Brussels, 2001
[3] Marek, P., Brozzetti, J., Guštar M., Ed.: Probabilistic Assessment of Structures using Monte Carlo Simulation: Background, Exercises, Software. ITAM CAS CZ Prague, 2001

[4] Marek, P., Krejsa, M.: Transition from Deterministic to Probabilistic Structural Steel Reliability Assessment with Special Attention to Stability Problems. SDSS '99, Sept. 1999, Timisoara, Romania

[5] Clough, R. W., Penzien, J.: Dynamics of Structures. Second edition. McGraw-Hill Book Company, New York, 1993

[6] Harris, C. M., Crede, C. E.: Shock and Vibration Handbook. Volume 3, McGraw-Hill Book Company, New York, 1961

Steven Vukazich, Associate Professor

phone: +4089243858

fax: +408924 4004

e-mail:vukazich@email.sjsu.edu

Department of Civil Engineering

San Jose State University

San Jose, CA

USA 95192-0083

Professor, Pavel Marek

e-mail: marekp@itam.cas.cz

ITAM Czech Academy of Sciences Czech Republic Prosecká 76, 19000 Praha 9, Czech Republic 